\title{
ON POSITIVE-DEFINITE INTEGRAL KERNELS AND A RELATED QUADRATIC FORM
}

\author{
BY \\ J. CHOVER AND J. FELDMAN
}

1. Introduction and preliminaries. Let $\rho$ be a continuous complex function of two real variables on the interval $J=[a, b]$. Let $C$ be the Banach space of continuous complex functions on $[a, b]$ with the norm

$$
\|f\|_{\infty}=\underset{a \leqq t \leqq b}{\text { l.u.b. }}|f(t)|,
$$

and $B$ the dual space of $C$. $B$ can be interpreted as the space of all complex Radon measures on $[a, b]$, where the measure $\mu$ acts on the function $f$ to give $\int f(t) d \mu(t)$ (all integrals are taken on $[a, b]$ unless otherwise stated), and the dual norm on $B$ can be interpreted as \|\|$\mu\|\|=$ total variation of $\mu$. A continuous operator $R$ from $B$ to $C$ is defined by $R \mu(t)=\int \rho(t, s) d \mu(s)$. We shall assume that $\rho$ is positive-definite, in the sense that $\iint \rho(s, t) d \mu(s) d \bar{\mu}(t)>0$ for all nonzero $\mu$ in $B$.

Let $H$ be $L_{2}[a, b]$ and let $S$ be the restriction of $R$ to those measures of the form $d m=h(s) d s, h \in H$. The function $\rho$ may also be interpreted as the covariance function of a Gaussian process defined with respect to: the probability space $\Omega$ of all complex functions on $J$, the Borel field generated by finite dimensional cylinder sets, and a measure $P$ induced by $\rho$ upon the latter. If $\rho$ satisfies certain mild differentiability conditions (Loeve, [4]; Doob, [2]), the outer measure $P^{*}$ of $C$ in $\Omega$ is unity, and both the measure and the stochastic process may be considered as defined on $C$. Thus the functions of $C$ may be considered as elements of a probability space. Statistical estimation theory has been applied to them (Grenander, [3]; Slepian, [5]). If one takes the values of such a function $f(t)$ at a finite sequence $\sigma$ of points $t_{1}^{\sigma}<t_{2}^{\sigma}<\cdots$ $<t_{n(\sigma)}^{\sigma}$ in $J$, and forms the expression

$$
\mu^{\sigma}(f)=\sum_{j=1}^{n(\sigma)} f\left(\hat{t}_{j}^{\sigma}\right) \bar{a}_{j}^{\sigma}(f)=\sum_{j=1}^{n(\sigma)} \sum_{k=1}^{n(\sigma)} \rho\left(t_{j}^{\sigma}, t_{k}^{\sigma}\right) a_{k}^{\sigma}(f) \tilde{a}_{j}^{\sigma}(f),
$$

where the $a_{j}^{\sigma}$ are defined by

$$
f\left(t_{k}^{\sigma}\right)=\sum_{j=1}^{n(\sigma)} \rho\left(t_{k}^{\sigma}, t_{j}^{\sigma}\right) a_{j}^{\sigma}(f),
$$

(existence and uniqueness being a consequence of the positive-definiteness of $\rho)$, then one has an essential component of an $n(\sigma)$-dimensional approxima-

Presented to the Society December 29, 1956; received by the editors February 23, 1957. 
tion to the "maximum-likelihood ratio" which one might wish to consider in estimating some parameter of the process.

Now from other considerations (Grenander, [3, pp. 221-222]) one can show that the set of functions $f$ for which the $\mu^{\sigma}(f)$ remain bounded as $\sigma$ is refined, is of $P$-measure zero. It is, however, of interest to characterize these functions analytically. We shall see that they are precisely the range of $S^{1 / 2}$ (Theorem 1).

We also shall investigate the range of $R$, that is, the collection of those $f$ in $C$ for which the equation $R \mu=f$ has a solution in $B$. Now, a simple necessary and sufficient condition that $f$ be in the range of $S$ is that

$$
\sum_{n=1}^{\infty} \frac{\left|f_{n}\right|^{2}}{\lambda_{n}^{2}}<\infty,
$$

where the $\lambda_{n}$ are the eigenvalues of $S$ (with proper multiplicity), i.e., $\lambda_{n} \phi_{n}(t)$ $=\int \rho(t, s) \phi_{n}(s) d s$ for some $\phi_{n}$ in $H$, and the $f_{n}$ are the Fourier coefficients $f_{n}=\int f(s) \bar{\phi}_{n}(s) d s$ with respect to a complete orthonormal set of eigenfunctions of $S$. (The special role played by Lebesgue measure on $J$ could be played equally well by any Radon measure whose support is the entire interval $J$; but if this measure is nonatomic, then a homeomorphism of $J$ will transform us back to the case of Lebesgue measure again.)

Of course, there is a necessary and sufficient condition for $f$ to be in the range of $R$, due to Hahn and Banach [1]: that there exist an $M>0$ such that

$$
\left|\sum_{k=1}^{n} f\left(t_{k}\right) \eta_{k}\right| \leqq M \max _{t \in J}\left|\sum_{k=1}^{n} \rho\left(t, t_{k}\right) \eta_{k}\right| \text {, }
$$

where the integer $n$ and the complex numbers $\eta_{1}, \eta_{2}, \cdots, \eta_{n}$ are arbitrary.

For many purposes, however, such criteria as the above are difficult to apply. In what follows, we approach the question otherwise: by examining the operators $S^{1 / 2}$ and $R^{1 / 2}$.

More notation: we shall write \|\| for the mean-square norm in $H$. It is well known that $S$ is a completely continuous, positive-definite operator on $H$. We note also that each eigenfunction is continuous, as is clear from the equation

$$
\lambda_{n} \phi_{n}(t)=\int \rho(t, s) \phi_{n}(s) d s
$$

and Mercer's theorem tells us that $\sum_{n} \lambda_{n} \phi_{n}(s) \Phi_{n}(t)$ converges uniformly to $\rho(s, t)$ (all sums are to run from 1 to $\infty$ unless otherwise indicated). The eigenfunctions are complete in $H$. Furthermore, their linear combinations are uniformly dense in $C$. For suppose this were not the case; then, by the Hahn-Banach theorem there would be some nonzero $\mu$ in $B$ with $\int \phi_{n}(t) d \mu(t)$ $=0$ for all $n$. But then 


$$
\begin{aligned}
\iint \rho(s, t) d \mu(s) d \bar{\mu}(t) & =\iint \sum_{n} \lambda_{n} \phi_{n}(s) \bar{\phi}_{n}(t) d \mu(s) d \bar{\mu}(t) \\
& =\sum \lambda_{n} \iint \phi_{n}(s) \phi_{n}(t) d \mu(s) d \bar{\mu}(t) \\
& =\sum \lambda_{n}\left|\int \phi_{n}(s) d \mu(s)\right|^{2}=0,
\end{aligned}
$$

contradicting the positive-definiteness assumption.

2. Convergence of approximating quadratic forms. We define $x(t)$, for $a \leqq t \leqq b$, as the element $\sum_{n} \phi_{n}(t)\left(\lambda_{n}\right)^{1 / 2} \phi_{n}$ in $H(x(t)$ is, for each $t$, a function on $[a, b])$; a legitimate definition, since $\sum_{n}\left|\bar{\phi}_{n}(t)\left(\lambda_{n}\right)^{1 / 2}\right|^{2}$ is finite, and, in fact, is just $\rho(t, t)$. Then clearly $(x(s), x(t))=\rho(t, s)$. Furthermore, the $x(t)$ span $H$; for $x(t) \phi_{n}(t)$ is a continuous function from $[a, b]$ to $H$, and if we take its Bochner integral, we get

$$
\begin{aligned}
\int x(t) \phi_{n}(t) d t & =\int \sum_{m}\left(\lambda_{m}\right)^{1 / 2} \bar{\phi}_{m}(t) \phi_{m} \phi_{n}(t) d t \\
& =\sum\left(\lambda_{m}\right)^{1 / 2} \phi_{m} \int \Phi_{m}(t) \phi_{n}(t) d t=\left(\lambda_{n}\right)^{1 / 2} \phi_{n} .
\end{aligned}
$$

We consider the $\mu^{\sigma}(f)$ of $\S 1$ :

$$
\mu^{\sigma}(f)=\sum_{j=1}^{n(\sigma)} f\left(t_{j}^{\sigma}\right) \tilde{a}_{j}^{\sigma}(f)=\sum_{j=1}^{n(\sigma)} \sum_{k=1}^{n(\sigma)} \rho\left(t_{j}^{\sigma}, t_{k}^{\sigma}\right) a_{k}^{\sigma}(f) \bar{a}_{j}^{\sigma}(f) .
$$

Then $\mu^{\sigma}(f)$ is clearly $\geqq 0$; let $\mu(f)$ be the l.u.b. (possibly infinite) of all $\mu^{\sigma}(f)$.

$K^{\sigma}$ is defined as the subspace of $H$ spanned by $x\left(t_{1}^{\sigma}\right), \cdots, x\left(t_{n(\sigma)}^{\sigma}\right)$, and $x^{\sigma}(f)$ is defined as $\sum_{\substack{n=1 \\ n(\sigma)}} a_{j}^{\sigma}(f) x\left(t_{j}^{\sigma}\right)$. Notice that $\mu^{\sigma}(f)$ is precisely $\left\|x^{\sigma}(f)\right\|^{2}$.

Lemma 1. If $\sigma \ll \tau$, then the projection of $x^{\tau}(f)$ on $K^{\sigma}$ is $x^{\sigma}(f)$.

Proof. We need only show that $x^{\tau}(f)-x^{\sigma}(f)$ is orthogonal to $K^{\sigma}$. Now since $\sigma \ll \tau$, there is for each $j$ from 1 to $n(\sigma)$ some $k(j)$ between 1 and $n(\tau)$ with $t_{j}^{\sigma}=t_{\boldsymbol{k}(j)}^{\tau}$. Then

$$
\begin{aligned}
\left(x^{\tau}(f)-x^{\sigma}(f), x\left(\dot{t}_{j}^{\sigma}\right)\right) & =\sum_{k=1}^{n(\tau)} a_{k}^{\tau}(f) \rho\left(t_{j}^{\sigma}, t_{k}^{\tau}\right)-\sum_{l=1}^{n(\sigma)} a_{l}^{\sigma}(f) \rho\left(\dot{t}_{j}^{\sigma}, t_{l}^{\sigma}\right) \\
& =\sum_{k=1}^{n(\tau)} a_{k}^{\tau}(f) \rho\left(t_{k(j)}^{\tau}, \dot{t}_{k}^{\tau}\right)-\sum_{l=1}^{n(\sigma)} a_{l}^{\sigma}(f) \rho\left(\tilde{t_{j}}, \dot{t_{l}}\right) \\
& =f\left(t_{k(j)}^{\tau}\right)-f\left(t_{j}^{\sigma}\right)=0 .
\end{aligned}
$$

For $g$ in $H$,

$$
S^{1 / 2} g(t)=\sum_{n}\left(\lambda_{n}\right)^{1 / 2}\left(g, \phi_{n}\right) \phi_{n}(t)
$$


Lemma 2. For any $g$ in $H$, the expansion for $S^{1 / 2} g$ in terms of the $\phi_{n}$ is uniformly convergent, and $S^{1 / 2}$ is a continuous map: $H \rightarrow C$.

Proof.

$$
\left|\sum_{n=n_{0}}^{\infty}\left(\lambda_{n}\right)^{1 / 2}\left(g, \phi_{n}\right) \phi_{n}(t)\right|^{2} \leqq\left(\sum_{n=n_{0}}^{\infty} \lambda_{n}\left|\phi_{n}(t)\right|^{2}\right)\left(\sum_{n=n_{0}}^{\infty}\left|\left(g, \phi_{n}\right)\right|^{2}\right),
$$

by the Schwartz inequality.

$$
\sum_{n=n_{0}}^{\infty} \lambda_{n}\left|\phi_{n}(t)\right|^{2} \leqq \rho(t, t),
$$

so the product goes to zero as $n$ increases, uniformly with respect to $t$. Further,

$$
\begin{aligned}
\left|\sum_{n}\left(\lambda_{n}\right)^{1 / 2}\left(g, \phi_{n}\right) \phi_{n}(t)\right|^{2} & \leqq \sum_{n} \lambda_{n}\left|\phi_{n}(t)\right|^{2} \sum_{n}\left|\left(g, \phi_{n}\right)^{2}\right| \\
& =\rho(t, t)\|g\|^{2} \rightarrow 0 \text { uniformly in } t \text { as }\|g\| \rightarrow 0 .
\end{aligned}
$$

THEOREM 1. If $\sigma \ll \tau$, then $\mu^{\sigma}(f) \leqq \mu^{\tau}(f)$. Furthermore $\mu(f)$ is finite if and only if $f=S^{1 / 2} g$ for some $g \in H$; and in this event, $\mu(f)=\|g\|^{2}$.

Proof. The first statement follows from Lemma 1 , since $\sigma \ll \tau$ implies $K^{\sigma}<K^{\tau}$, so that $\mu^{\sigma}(f)=\left\|x^{\sigma}(f)\right\|^{2} \leqq\left\|x^{\tau}(f)\right\|^{2}=\mu^{\tau}(f)$.

Next suppose $f=S^{1 / 2} g$ for some $g$ in $H$. Then $\left(f, \phi_{n}\right)=\left(\lambda_{n}\right)^{1 / 2}\left(g, \phi_{n}\right)$. Let $\gamma_{n}=\left(g, \phi_{n}\right)$. Then $(g, x(t))=\left(\sum_{n} \gamma_{n} \phi_{n}, \sum_{n} \phi_{n}(t)\left(\lambda_{n}\right)^{1 / 2} \phi_{n}\right)=\sum_{n}\left(f, \phi_{n}\right) \phi_{n}(t)$. The map $t \rightarrow x(t)$ is continuous from $[a, b]$ to $H$; therefore $(g, x(t))$ is continuous in $t$. But the equality just derived shows that it equals $f(t)$ almost everywhere; therefore, since $f$ is continuous, $(g, x(t))=f(t)$ for all $t$ in $[a, b]$. Then, denoting by $P_{\sigma}$ the projection operator from $H$ onto $K^{\sigma}$, we get $\left(P_{\sigma} g, x\left(t_{j}^{\sigma}\right)\right)=\left(g, x\left(t_{j}^{\sigma}\right)\right)=f\left(t_{j}^{\sigma}\right)=\left(x^{\sigma}(f), x\left(t_{j}^{\sigma}\right)\right)$, so that $P_{\sigma} g=x^{\sigma}(f)$, and $\mu^{\sigma}(f)$ $=\left\|x^{\sigma}(f)\right\|^{2}=\left\|P_{\sigma} g\right\|^{2} \leqq\|g\|^{2}$. In fact, $\mu(f)=\|g\|^{2}$, because $U_{\sigma} K^{\sigma}$ is dense in $H$, so that, as $\sigma$ gets finer and finer, $P_{\sigma}$ converges strongly to the identity operator in $H$, and therefore $\left\|P_{\sigma} x\right\|^{2} \rightarrow\|x\|^{2}$.

On the other hand, suppose $\mu(f)$ is finite. Then $\left\|x^{\sigma}(f)\right\|^{2}$ is bounded, so that, by the weak compactness of a sphere in Hilbert space, there is some $g$ in $H$ and some cofinal sequence $\left\{\sigma_{\alpha}\right\}$ such that $x^{\sigma_{\alpha}}(f)$ converges weakly to $g$. Then, for any $y$ in $K^{\sigma}$, we have

$$
\begin{aligned}
\left(P_{\sigma} g, y\right) & =(g, y)=\lim _{\alpha}\left(x^{\sigma}(f), \dot{y}\right) \\
& =\lim _{\alpha}\left(P_{\sigma} x^{\sigma}(f), y\right)=\lim _{\alpha}\left(x^{\sigma}(f), y\right)=\left(x^{\sigma}(f), y\right), \text { so that } P_{\sigma} g=x^{\sigma}(f) .
\end{aligned}
$$

Now denote by $t^{\sigma}$ the closest point of $\sigma$ to $t$. Then

$$
\begin{aligned}
\left|\left(x^{\sigma}(f), x(t)\right)-f(t)\right| \leqq & \left|\left(x^{\sigma}(f), x\left(t^{\sigma}\right)\right)-f\left(t^{\sigma}\right)\right| \\
& +\left\|x^{\sigma}(f)\left|\left\||| x\left(t^{\sigma}\right)-x(t)\right\|+\right| f\left(t^{\sigma}\right)-f(t) \mid .\right.
\end{aligned}
$$


$f\left(t^{\sigma} \alpha\right) \rightarrow f(t)$ as $\alpha$ increases, and similarly $x\left(t^{\sigma}\right) \rightarrow x(t) .\left\|x^{\sigma}(f)\right\|$ is bounded by $(\mu(f))^{1 / 2}$. And $\left(x^{\sigma}(f), x\left(t^{\sigma}\right)\right)$ is precisely $f\left(t^{\sigma}\right)$. Therefore $(g, x(t))=f(t)$. But write $g$ in the form $\sum_{n-1}^{\infty} \gamma_{n} \phi_{n}$. Then

$$
(g, x(t))=\left(\sum_{n} \gamma_{n} \phi_{n}, \sum_{m=1}^{\infty} \phi_{m}(t)\left(\lambda_{m}\right)^{1 / 2} \phi_{m}\right)=\sum_{n} \gamma_{n}\left(\lambda_{n}\right)^{1 / 2} \phi_{n}(t) .
$$

Thus, we have $f=S^{1 / 2} g$. Alternately phrased, $\mu(f)<\infty$ if and only if

$$
\sum_{n} \frac{\left|\left(f, \phi_{n}\right)\right|^{2}}{\lambda_{n}}<\infty .
$$

REMark 1. Considering $f$ now as an element of a probability space $C$, we may interpret $\psi_{n}(f)=\lambda_{n}^{-1 / 2}\left(f, \phi_{n}\right)=\lambda_{n}^{-1 / 2} f_{n}$ as random variables, which are easily seen to be Gaussian. Furthermore,

$$
\begin{aligned}
\lambda_{n}^{-1 / 2} \lambda_{m}^{-1 / 2} \int_{C} f_{n} \bar{f}_{m} d P(f) & =\lambda_{n}^{-1 / 2} \lambda_{m}^{-1 / 2} \int_{J} d s \int_{J} d t \phi_{n}(s) \bar{\phi}_{m}(t) \int_{C} f(s) \bar{f}(t) d P(f) \\
& =\lambda_{n}^{-1 / 2} \lambda_{m}^{-1 / 2} \int_{J} d s \int_{J} d t \rho(t, s) \phi_{n}(s) \phi_{m}(t)=\delta_{m n}
\end{aligned}
$$

Thus the $\psi_{n}(f)$ are uncorrelated, and, since Gaussian, therefore independent. They all have variance equal to unity. By the standard convergence theorems it is then easy to conclude the fact mentioned in $\$ 1$, namely, that the set of $f$ for which $\sum_{n}\left|\psi_{n}(f)\right|^{2}<\infty$ is a set of zero probability.

REMARK 2 . We are also in a position to say something about the dependence of $\mu(f)$ upon the interval $J$. In fact, let $J^{\prime}$ be any subinterval of $J$ with nonempty interior; define eigenvalues and eigenfunctions

$$
\lambda_{n}^{\prime} \phi_{n}^{\prime}(t)=\int_{J^{\prime}} \rho(t, s) \phi_{n}^{\prime}(s) d s, \quad t \in J^{\prime}
$$

and let $\mu_{J^{\prime}}(f)$ be defined by restricting $\sigma$ to $J^{\prime}$. Then we have

THEOREM 2. $\mu_{J^{\prime}}(f) \leqq \mu(f)$; so that if $\mu(f)<\infty$ then also $\mu_{J^{\prime}}(f)<\infty$ and hence

$$
\sum_{n} \frac{\left|\left(f, \phi_{n}^{\prime}\right)^{\prime}\right| 2}{\lambda_{n}^{\prime}}<\infty,
$$

where

$$
\left(f, \phi_{n}^{\prime}\right)^{\prime}=\int_{J^{\prime}} f(t) \Phi_{n}^{\prime}(t) d t .
$$

Proof. The first statement follows from Lemma 1; the second, by considering the arguments for Theorem 1 rephrased in terms of $L_{2}\left(J^{\prime}\right)$.

REMARK 3. If $K_{t}$ is the closed linear manifold in $H$ spanned by the $x(s)$, 
$a \leqq s \leqq t \leqq b$, and if $\pi_{t}$ is the projection onto $K_{t}$, then $\mu_{[a, t]}(f)=\left\|\pi_{t} g\right\|^{2}$, where $f=S^{1 / 2} g, g \in H$. The $\pi_{t}$ form a resolution of the identity operator on $H$, and $\left\|\pi_{t}(g)\right\|$ is a left-continuous (and, of course, monotonic nondecreasing) function of $t$. That it is continuous on the left for each $t$ in $J$ follows from the continuity of $\rho$ : firstly, for $s \geqq s_{0},\left\|\pi_{8} x\left(s_{0}\right)\right\|$ is constant; and as $s \uparrow s_{0},\left\|\pi_{8} x\left(s_{0}\right)\right\|$ $\uparrow\left\|\pi_{s_{0}} x\left(s_{0}\right)\right\|$, since

$$
\begin{aligned}
\left|\rho\left(s_{0}, s\right)\right| & =\left|\left(x\left(s_{0}\right), x(s)\right)\right|=\left|\left(\pi_{8} x\left(s_{0}\right), x(s)\right)\right| \\
& \leqq\left\|\pi_{8} x\left(s_{0}\right)\right\|\|x(s)\| \leqq\left\|x\left(s_{0}\right)\right\|\|x(s)\|
\end{aligned}
$$

and the extreme members of the above chain both approach $\left\|x\left(s_{0}\right)\right\|^{2}$ as $s \uparrow s_{0}$. Let now $g$ be any element in $H$. Given $\epsilon>0$, one can find a finite linear combination $\sum_{1}^{n} a_{i} x\left(s_{i}\right)$ with $a \leqq s_{i} \leqq s_{0}$ such that $\left\|\sum_{i}^{n} a_{i} x\left(s_{i}\right)-\pi_{s_{0}} g\right\|<\epsilon / 2$. If one then picks $\delta$ so small that $\left\|\pi_{s} x\left(s_{i}\right)-\pi_{s_{0}} x\left(s_{i}\right)\right\|<\left[\sum\left|a_{i}\right|^{-1}\right] \epsilon / 2$ for $\left|s-s_{0}\right|<\delta$ and for each $i=1,2, \cdots, n$; one can insure that $\left\|\left(\pi_{s}-\pi_{s_{0}}\right) \pi_{s_{0} g}\right\|$ $=\left\|\pi_{s} g-\pi_{s_{0}} g\right\|<\epsilon$, and thus show the left-continuity of $\left\|\pi_{s} g\right\|$ at $s_{0}$.

REMARK 4. If $\rho^{\prime}$ is another kernel satisfying the same assumptions as $\rho$, $S^{\prime}$ the corresponding operator on $H$, and $\mu^{\prime \sigma}(f)$ the corresponding approximating sums, and if it happens that $S^{\prime} \geqq c S$ for some constant $c>0$, then boundedness of the $\mu^{\sigma}(f)$ for any particular $f$ implies boundedness of the $\mu^{\prime \sigma}(f)$. For example, let

$$
\rho(s, t)=\int_{-\infty}^{\infty} e^{i(\sigma-t)} d F(\lambda)
$$

and

$$
\rho^{\prime}(s, t)=\int_{-\infty}^{\infty} e^{i(\Omega-t)} d F^{\prime}(\lambda), \quad \quad d F^{\prime} \geqq c d F .
$$

The truth of the remark follows from the next lemma.

Lemma 3. Let $A, B$ be bounded operators on $H, c$ a positive constant, and $A \geqq c B \geqq 0$. Then the range of $A^{1 / 2}$ contains that of $B^{1 / 2}$.

Proof. Define $T_{0}$ from the range of $A^{1 / 2}$ to that of $B^{1 / 2}$ by $T_{0}\left(A^{1 / 2} x\right)$ $=B^{1 / 2} x$. Since $c^{2}\left\|B^{1 / 2} x\right\|^{2} \leqq\left\|A^{1 / 2} x\right\|^{2}$, this definition makes sense, and, in fact, $T_{0}$ is bounded by $c^{-1}$. $T_{0}$ can therefore be extended to a bounded operator $T$ on all of $H$. Now, $T A^{1 / 2}=B^{1 / 2}$, so $A^{1 / 2} T^{*}=B^{1 / 2}$, and the range of $A^{1 / 2}$ clearly contains that of $B^{1 / 2}$.

3. The operator $R^{1 / 2}$. One can define a square root for $R$, as follows: given $\mu$ in $B$, let $\gamma_{n}=\left(\lambda_{n}\right)^{1 / 2} \int \Phi_{n}(s) d \mu(s)$. Then

$$
\sum_{n}\left|\gamma_{n}\right|^{2}=\sum \lambda_{n} \iint \Phi_{n}(s) \phi_{n}(t) d \mu(s) d \bar{\mu}(t)=\iint \rho(t, s) d \mu(s) d \bar{\mu}(t),
$$

and we define $R^{1 / 2} \mu$ to be the element $\sum_{n} \gamma_{n} \phi_{n}$ in $H$. So $R^{1 / 2}$ is a linear map from $B$ to $H$. Let $B_{0}$ denote the unit sphere of $B$. 
Lemma 4. $R$ is continuous from $B_{0}$ in the weak topology to $C$ in the norm topology (and hence also from $B$ in the norm topology to $C$ in the norm topology).

Proof. If $\mu_{\alpha}$ is weakly convergent to $\mu$, and $\mu_{\alpha}, \mu \in B_{0}$, then $\mu_{\alpha}-\mu \in 2 B_{0}$ and $\mu_{\alpha}-\mu$ converges weakly to zero. So it will suffice to consider the case where $\mu_{\alpha} \in B_{0}$ and $\mu_{\alpha}$ converges weakly to zero.

$$
\begin{aligned}
\left|\int \rho(s, t) d \mu_{\alpha}(t)\right| \leqq & \sum_{n=1}^{n_{0}} \lambda_{n}\left\|\phi_{n}\right\|_{\infty}\left|\int \Phi_{n}(t) d \mu_{\alpha}(t)\right| \\
& +\left|\int \sum_{n=n_{0}+1}^{\infty} \lambda_{n} \phi_{n}(s) \Phi_{n}(t) d \mu_{\alpha}(t)\right| .
\end{aligned}
$$

By choosing $n_{0}$ large, we can make

$$
\left|\sum_{n=n_{0}+1}^{\infty} \lambda_{n} \phi_{n}(s) \Phi_{n}(t)\right|<\epsilon / 2
$$

so that

$$
\left|\int \rho(s, t) d \mu_{\alpha}(t)\right| \leqq \sum_{n=1}^{n_{0}} \lambda_{n}\left\|\phi_{n}\right\|_{\infty} \int \bar{\phi}_{n}(t) d \mu_{\alpha}(t)+\epsilon / 2 \mid\left\|\mu_{\alpha}\right\| \| .
$$

Then, for sufficiently large $\alpha$, we have

$$
\sum_{n=1}^{n_{0}} \lambda_{n}\left\|\phi_{n}\right\|_{\infty}\left|\int \Phi_{n}(t) d \mu_{\alpha}(t)\right|<\epsilon / 2,
$$

so that $\left|\int \rho(s, t) d \mu_{\alpha}(t)\right|<\epsilon$ for such $\alpha$.

TheOREM 3. $R^{1 / 2}$ is continuous from $B_{0}$ in the weak topology to $H$ in the norm topology (and hence also from $B$ in the norm topology to $H$ in the norm topology). The equality

$$
\left(h, R^{1 / 2} \mu\right)=\int S^{1 / 2} h(s) d \bar{\mu}(s)
$$

holds for all $h \in H$ and $\mu \in B$. If $d_{\mu}$ is of the form gds with $g$ in $H$, then $S^{1 / 2} g$ $=R^{1 / 2} \mu$. And finally, $S^{1 / 2} R^{1 / 2}=R^{1 / 2} R^{1 / 2}=R$.

Proof. As in proving Lemma 4 , it suffices in the continuity proof to show that if $\mu_{\alpha}$ converges weakly to 0 while \|\|$\mu_{\alpha} \| \mid$ remains bounded by 1 , then $R^{1 / 2} \mu_{\alpha}$ converges in norm to zero. Now,

$$
\begin{aligned}
\left\|R^{1 / 2} \mu_{\alpha}\right\|^{2} & =\sum_{n} \lambda_{n}\left|\int \Phi_{n}(s) d \mu_{\alpha}(s)\right|^{2} \\
& =\sum_{n=1}^{n_{0}} \lambda_{n}\left|\int \Phi_{n}(s) d \mu_{\alpha}(s)\right|^{2}+\iint \sum_{n=n_{0}+1}^{\infty} \lambda_{n} \bar{\phi}_{n}(s) \phi_{n}(t) d \mu(s) d \bar{\mu}(t),
\end{aligned}
$$


and, proceeding as in Lemma 4 , we get this small by first choosing $n_{0}$ large and then choosing $\alpha$ large.

The equality $\left(h, R^{1 / 2} \mu\right)=\int S^{1 / 2} h(s) d \bar{\mu}(s)$ can be seen as follows: in Lemma 2 it was pointed out that $\sum_{n}\left(\lambda_{n}\right)^{1 / 2}\left(h, \phi_{n}\right) \phi_{n}(t)$ converges uniformly. Then $\int \sum_{n}\left(\lambda_{n}\right)^{1 / 2}\left(h, \phi_{n}\right) \phi_{n}(s) d \bar{\mu}(s)=\sum_{n} \gamma_{n}\left(h, \phi_{n}\right)$, where $R^{1 / 2} \mu=\sum \gamma_{n} \phi_{n}$; this is the desired result.

If $d \mu=g d t$, then $\left(S^{1 / 2} g, \phi_{n}\right)=\left(\lambda_{n}\right)^{1 / 2} \int \Phi_{n}(s) g(s) d s$, but also $\left(R^{1 / 2} \mu, \phi_{n}\right)$ $=\left(\lambda_{n}\right)^{1 / 2} \int \Phi_{n}(s) d \mu(s)$, so that $S^{1 / 2} g=R^{1 / 2} \mu$.

To see that $S^{1 / 2} R^{1 / 2}=R$ : first, observe that $S^{1 / 2} R^{1 / 2}\left|H=S^{1 / 2} S^{1 / 2}\right| H=S$ $=R \mid H$. Now, by Lemma 2 and the first part of this theorem, $S^{1 / 2} R^{1 / 2}$ is continuous from $B_{0}$ in the weak topology to $C$ in the norm topology. Lemma 4 asserts the same for $R$. Finally, $H \cap B_{0}$ is weakly dense in $B_{0}$, since in particular the finite linear combinations of characteristic functions of Borel sets are so. Therefore $S^{1 / 2} R^{1 / 2}$ and $R$ agree on $B_{0}$, and hence on all of $B$.

Corollary. If $f=S^{1 / 2} g, g \in H$, and also $f=R \mu, \mu \in B$, then $\int f(s) d \bar{\mu}(s)$ $=\|g\|^{2}$.

THEOREM 4. The range of $R$ is a proper subset of the range of $S^{1 / 2}$.

Proof. That it is a subset is clear from the equality $S^{1 / 2} R^{1 / 2}=R$. To see that it is a proper subset: the image under $R^{1 / 2}$ of $B_{0}$ is a compact subset of $H$, since $B_{0}$ is weakly compact. Now, the range of $R$ is the image under $S^{1 / 2}$ of the range of $R^{1 / 2}$. Since $S^{1 / 2}$ has no nullspace, its range would thus equal that of $R$ only if the range of $R^{1 / 2}$ were all of $H$. But $R^{1 / 2}\left(B_{0}\right)$ is compact in $H$, hence nowhere dense. Then $\bigcup_{n=1}^{\infty}\left(R_{0}\right)^{1 / 2}\left(n B_{0}\right)$ is a first category set in $H$, and certainly not all of $H$.

\section{BIBLIOGRAPHY}

1. S. Banach, Theorie des operation linéaires, Warsaw, 1932, Chapter IV, §2.

2. J. L. Doob, Stochastic processes depending on a continuous parameter, Trans. Amer. Math. Soc. vol. 42 (1937) pp. 107-110.

3. U. Grenander, Stochastic processes and statistical inference, Ark. Mat. vol. 1, no. 17 (1950) pp. 195-277.

4. M. Loève (a note by) in P. Levy, Processus stochastiques et mouvement Brownien, Paris 1948 , p. 316.

5. D. Slepian, Estimation of signal parameters in the presence of noise, Trans. IRE Professional Gp. on Information Theory, PGIT-3, March 1954, pp. 68-89.

UNIVERSITY OF WisCoNSIN,

MAdison, Wis.

Bell Telephone laboratories,

NEW YoRK, N. Y.

Columbia University,

NEW YoRK, N. Y. 